\title{
SREBP2 is upregulated in esophageal squamous cell carcinoma and co-operates with c-Myc to regulate HMGCR expression
}

\author{
CHENXI ZHONG ${ }^{*}$, LIMIN FAN*, ZHIGANG LI, FENG YAO and HENG ZHAO \\ Department of Thoracic Surgery, Shanghai Chest Hospital, Shanghai Jiao Tong University, Shanghai 200030, P.R. China
}

Received December 31, 2017; Accepted October 1, 2018

DOI: $10.3892 / \mathrm{mmr} .2019 .10577$

\begin{abstract}
Dysregulations of the mevalonate pathway (MVA) have been previously identified. Our previous study demonstrated that 3-hydroxy-3-methylglutaryl-coenzyme A reductase (HMGCR), the rate-limiting enzyme of the MVA pathway, was upregulated in esophageal squamous cell carcinoma (ESCC) and statin-inhibited ESCC tumorigenesis. However, the underlying mechanism of HMGCR regulation in ESCC remains unknown. In the present study, western blotting and immunohistochemistry analysis demonstrated that sterol regulatory element-binding protein 2 (SREBP2), the master regulator for HMGCR, was upregulated in ESCC clinical samples. Overexpression of SREBP2 expression in ESCC cell lines promoted the growth, migration and colony formation of cancer cells in the MTT, Boyden chamber and soft agar assays, respectively, which was inhibited by lovastatin. Downregulation of SREBP2 expression in ESCC cell lines inhibited the viability, and migration and colony formation abilities of cancer cells. Assessment of the molecular mechanism demonstrated that SREBP2 interacted with c-Myc and cooperated with c-Myc to activate HMGCR expression. Collectively, the present study identified SREBP2 as an oncogene associated with the tumorigenesis of ESCC and further demonstrated the therapeutic effects of statins in ESCC.
\end{abstract}

\section{Introduction}

Esophageal squamous cell carcinoma (ESCC) is one of the most common types of malignancies in China (1). Significant advances in the diagnosis of ESCC have been made; however, the outcome of ESCC remains poor (1). Dysregulations in

Correspondence to: Dr Feng Yao or Dr Heng Zhao, Department of Thoracic Surgery, Shanghai Chest Hospital, Shanghai Jiao Tong University, 241 West Huaihai Road, Shanghai 200030, P.R. China

E-mail: yaofeng20120913@aliyun.com

E-mail: shchestsurgery@163.com

${ }^{*}$ Contributed equally

Key words: sterol regulatory element-binding protein 2, esophageal squamous cell carcinoma, c-Myc, statin metabolism are one of the hallmarks of cancer cells $(2,3)$. An improved understanding of the molecular mechanisms underlying the dysregulated metabolism in cancer cells may aid developments in the treatment of ESCC.

The mevalonate (MVA) pathway is responsible for the de novo synthesis of cholesterol $(4,5)$. The 3-hydroxy 3-methylglutaryl (HMG)-coenzyme A reductase (HMGCR) is the rate-limiting enzyme of cholesterol synthesis and is regulated via a negative feedback mechanism mediated by sterols and non-sterol metabolites derived from MVA $(6,7)$. Statins inhibit the activity of HMGCR, which is regulated by sterol regulatory element-binding protein 2 (SREBP2) $(8,9)$. A lack of intracellular sterols leads to the translocation of SREBP2 from the endoplasmic reticulum to the Golgi; SREBP2 is cleaved by proteases in the Golgi membrane $(10,11)$. The N-terminal of SREBP2 serves as a transcription factor and enters the nucleus to activate the transcription of enzymes involved in cholesterol biosynthesis $(10,11)$.

In our previous study, it was demonstrated that the expression of enzymes involved in the MVA pathway was upregulated in ESCC clinical tissues, and treatment with statin inhibited the growth and tumorigenesis of ESCC cells (12). Additionally, it was demonstrated that HMGCR promoted the growth, migration and colony formation of ESCC cells (13); however, whether SREBP2 associates with other factors to regulate HMGCR expression in ESCC cells remains unknown.

In the present study, the expression profile and biological functions of SREBP2 in ESCC cells were investigated and an SREBP2-binding protein was identified. In addition, the underlying molecular mechanisms of SREBP2 in ESCC were examined.

\section{Materials and methods}

Cell culture and clinical samples. A normal human esophageal cell line (Het-1A) was obtained from the American Type Culture Collection (Manassas, VA, USA). 293T, Eca109 and KYSE150 cells were obtained from the cell bank (the Chinese Academy of Science, Shanghai, China). The Caes17 and KYSE180 cells were gifts from Professor Xu (Shantou Medical University, Shantou, China) (14). Cells were cultured in Dulbecco's modified Eagle's medium (DMEM; Invitrogen; Thermo Fisher Scientific, Inc., Waltham, MA, USA) supplemented with $10 \%$ fetal bovine serum (Thermo Fisher Scientific, Inc.), penicillin $(100 \mathrm{U} / \mathrm{ml})$ and streptomycin $(100 \mu \mathrm{g} / \mathrm{ml})$. 
Cell cultures were maintained in a humidified incubator with $5 \% \mathrm{CO}_{2}$ at $37^{\circ} \mathrm{C}$.

Patient sample collection (esophageal cancer and adjacent non-tumor samples) was approved by the Institutional Ethics Committee of Shanghai Chest Hospital (Shanghai, China), and all patients provided written informed consent. A total of 52 patients (32 males and 20 females, 45-78 years old with middle and lower esophageal cancer) between May 12010 and June 242014 who had not undergone radiotherapy and/or chemotherapy were recruited for the present study.

Reverse transcription-quantitative polymerase chain reaction $(R T-q P C R)$. Total RNA was extracted from tissue or cell samples with TRIzol ${ }^{\circledR}$ (Thermo Fisher Scientific, Inc.) and cDNA was synthesized using an MMLV Reverse Transcription kit (Promega Corporation, Madison, WI, USA) at $37^{\circ} \mathrm{C}$ for $1 \mathrm{~h}$. qPCR was performed with $2 X$ SYBR Green mixture (Takara Biotechnology Co., Ltd., Dalian, China) in triplicate. The thermocycling conditions were: $95^{\circ} \mathrm{C}$ for $2 \mathrm{~min}$; then 45 cycles of $94^{\circ} \mathrm{C}$ for $15 \mathrm{sec}, 55^{\circ} \mathrm{C}$ for $15 \mathrm{sec}$ and $68^{\circ} \mathrm{C}$ for $30 \mathrm{sec}$. The relative expression levels of target genes were normalized to $18 \mathrm{~S}$ rRNA (Applied Biosystems; Thermo Fisher Scientific, Inc.) and were calculated using $2^{-\Delta \Delta \mathrm{Cq}}(15)$. The primer sequences for SREBP2 were: F, 5'-TGACTTGTTTTCAGAACAGC-3' and R, 5'-AACTCTGCAAGTCAAGGTT-3'.

Immunohistochemistry. ESCC tissues and paired non-cancerous tissues were fixed with $4 \%$ PFA overnight at $4^{\circ} \mathrm{C}$ for $8 \mathrm{~h}$ and embed in paraffin wax. The paraffin-embed ESCC sections (5- $\mu \mathrm{m}$-thick) were kept at $65^{\circ} \mathrm{C}$ for $30 \mathrm{~min}$ and then deparaffinized, washed in with xylene and rehydrated with descending ethanol series (100, 95, 75 and 50\%). Endogenous peroxidase activity was blocked with $0.35 \% \mathrm{H}_{2} \mathrm{O}_{2}$ solution. Antigen retrieval was performed using citrate solution $(10 \mathrm{mM}, \mathrm{pH} 6.0)$ at $98^{\circ} \mathrm{C}$ for $20 \mathrm{~min}$. Non-specific binding was blocked with $1 \%$ bovine serum albumin solution (Sangon Biotech Co., Ltd., Shanghai, China) at room temperature for $1 \mathrm{~h}$. Sections were stained with an SREBP2 antibody (1:100; HPA031962; Sigma-Aldrich; Merck $\mathrm{KGaA}$, Darmstadt, Germany) at $4^{\circ} \mathrm{C}$ for $8 \mathrm{~h}$. Then, the sections were washed with TBST and incubated with the secondary antibody [Gene Tech (Shanghai) Co., Ltd., Shanghai, China] for $2 \mathrm{~h}$ at room temperature. Following washing with TBST three times ( $5 \mathrm{~min}$ for each time), the sections were developed with 3,3'-diaminobenzidine ( $2 \mathrm{~min}$ at room temperature) and counterstained with hematoxylin ( $8 \mathrm{~min}$ at room temperature). The sections were examined under an inverted microscope and images acquired at the magnification, $\mathrm{x} 20$.

Western blotting. Cellular proteins were harvested from tissues and cells with radioimmunoprecipitation assay buffer (Thermo Fisher Scientific, Inc.) containing a protease inhibitor (Sigma-Aldrich; Merck KGaA). Cell lysates were placed on ice for $30 \mathrm{~min}$ and were subsequently centrifuged at 12,000 $\mathrm{x}$ g for $10 \mathrm{~min}$ at $4^{\circ} \mathrm{C}$. The Bradford method was used to determine the protein concentration. Equal amounts of proteins (20 $\mu \mathrm{g} / \mathrm{lane})$ were loaded to $10 \%$ SDS-PAGE and were transferred to a polyvinylidene difluoride membranes. Subsequently, the membranes were blocked (5\% non-fat milk in $1 \mathrm{X}$ Tris-buffered saline with Tween-20) for $1 \mathrm{~h}$ at room temperature. The membranes were incubated with primary antibodies overnight at $4^{\circ} \mathrm{C}$ : SREBP2
(1:1,000; ab30682; Abcam, Cambridge, UK), c-Myc (1:1,000; 12189; Cell Signaling Technology, Inc., Danvers, MA, USA), HMGCR (1:1,000; HPA008338; Sigma-Aldrich; Merck KGaA) and GAPDH (1:5,000; EMD Millipore, Billerica, MA, USA). Secondary antibodies conjugated with peroxidase $(1: 1,000$; 14708 and 4878; Cell Signaling Technology, Inc.) were applied to membranes for $1 \mathrm{~h}$ at room temperature. Following immunoblotting, the films were scanned with a Bio-Rad imaging system (Bio-Rad Laboratories, Inc., Hercules, CA, USA).

Mass spectrometry assay. The coding sequence for SREBP2 was inserted into pCMV14/3xFLAG-Pax3 (25427; Adgene, Watertown, MA, USA) using the restriction enzymes EcoR1 and EcoRV. Subsequently, the plasmids (Flag-SREBP2) were transfected into 293T cells (exogenous protein is highly expressed in this cell line) using Lipofectamine ${ }^{\circledR} 2000$ (Invitrogen; Thermo Fisher Scientific, Inc.). The transfection efficiency was monitored by the co-transfecting GFP. After $48 \mathrm{~h}$, the cells were harvested, and immunoprecipitation was performed as described below. The immunoprecipitates were subjected to SDS-PAGE and stained with silver solution at room temperature for $1 \mathrm{~h}$. The differentially expressed bands were collected and digested with trypsin followed by mass spectrometry analysis (PTM Biolabs Inc., Hangzhou, China; http://www.ptm-biolab.com.cn).

Plasmids and cell transfection. The SREBP2 sequence was generated by PCR using KYSE180 cDNA and subcloned into the pCMVTag2B vector (11463; Adgene). KOD polymerase (Takara Biotechnology Co., Ltd.) was used. The primer sequences for SREBP2 were: F, 5'-ATGGACGACAGCGGC GAG-3' and R, 5'-TCAGGAGGCGGCAATGGC-3'. The c-Myc sequence was generated by PCR and subcloned into the pcDNA3.1 vector (containing HA tag). Caes17 and KYSE180 cells were transfected with SREBP2 plasmids (1 $\mu \mathrm{g})$ using Lipofectamine ${ }^{\circledR} 2000$ (Invitrogen; Thermo Fisher Scientific, Inc.) according to the manufacturer's protocol. After $48 \mathrm{~h}$, transfected cells were selected using G418 $(600 \mu \mathrm{g} / \mathrm{ml})$ for 2 weeks. After 2 weeks, G418-resistant cells were pooled.

Boyden chamber assays. Caes17 and KYSE180 cells (1x10 $10^{5}$ cells/well) were seeded on the top layer of the chamber. Medium supplemented with serum was used as a chemoattractant in the bottom chamber for the migration assay. The cells were incubated at $37^{\circ} \mathrm{C}$ for $8 \mathrm{~h}$. The same protocol was used for the invasion assay; however, the membrane was pre-coated with $50 \mu \mathrm{g}$ Matrigel. The non-migratory cells in the top chambers were removed with cotton swabs. The migrated and invaded cells on the lower membrane surface were fixed in $100 \%$ methanol for $5 \mathrm{~min}$ at room temperature, air-dried, stained with eosin for $5 \mathrm{~min}$ at room temperature and subsequently counted under an inverted microscope (magnification, $\mathrm{x} 20$ ).

Cell viability assay. An MTT assay was performed to evaluate the viability of the cells. Cells were counted and placed in 96-well plates (1,000 cells/well) in triplicate and incubated with fresh medium. At $4 \mathrm{~h}$ prior to the analysis of cell viability at the indicated time points (day 2, 4, 6 and 8), cells were incubated with MTT (200 $\mu \mathrm{l} / \mathrm{ml}$ medium) for $4 \mathrm{~h}$. Following the MTT 
incubation, the formazan crystals were dissolved using DMSO. Subsequently, growth curves were generated by analyzing the optical density at $540 \mathrm{~nm}$ using a microplate reader.

Soft agar assay. Caes17 and KYSE180 cells (1x10³ cells /well) were seeded in $1 \mathrm{ml} 0.5 \%$ noble agar in complete DMEM overlaid with $2 \mathrm{ml} 1 \%$ agar in the same medium. After 2 weeks of culture, cell colonies were examined under a microscope.

SREBP2, HMGCR and c-Myc knockdown in ESCC cell lines. SREBP2, HMGCR and c-Myc expression in ESCC cell lines was downregulated via lentiviral transduction. The lentivirus was purchased from Shanghai GeneChem Co., Ltd. (Shanghai, China). Cells were seeded in 6 -well plates $\left(5 \times 10^{5}\right.$ cells/well) and incubated with the lentivirus for $24 \mathrm{~h}$. The lentivirus expressed GFP independent of siRNA. GFP-positive cells were sorted by cytometry. The sequences for the siRNAs are: Si con: 5'-AATCTGAGTCCTTGCTTAATG-3', Si SREBP2 1\#: 5'-AATCAAGTGGGAGAGTTCCCT-3', Si SREBP2 2\#: 5'-AAGCTAAGACCAGCGCCCGGG-3', Si HMGCR 1\#: 5'-AAGTCATAGTGGGGACAGTGA-3', SiHMGCR 2\#: 5'-AACCAGAAATGTGATTCAGTA-3', Si c-Myc 1\#: 5'-AAC GTTAGCTTCACCAACAGG-3' and Si c-Myc 2\#: 5'-AAC CAGAGTTTCATCTGCGAC-3'.

Glutathione S-transferase (GST) pull-down assay. The GST-Myc fusion protein was purified using GST Protein Interaction Pull-Down kit (21516; Pierce; Thermo Fisher Scientific, Inc.). Caes17 cells were harvested using RIPA buffer. Then, the cell lysates were centrifugated $\left(12,000 \mathrm{x} \mathrm{g}\right.$ at $4^{\circ} \mathrm{C}$ for $20 \mathrm{~min}$ ). Subsequently, $10 \mu \mathrm{g}$ GST-Myc protein was incubated with the lysates of Caes 17 cells at $4^{\circ} \mathrm{C}$ overnight. Sepharose $4 \mathrm{~B}$ beads were added to the supernatant and incubated for $4 \mathrm{~h}$ at $4^{\circ} \mathrm{C}$. Protein binding to GST-Myc was immunoprecipitated and analyzed by $8 \%$ SDS-PAGE with an anti-GST antibody (1:5,000, ab19256, Abcam). The blots were examined using a Bio-Rad imaging system.

HMGCR promoter assay. The promoter region (-2000bp-1bp) of HMGCR was cloned into pGL3 basic vectors using Mlu I and $\mathrm{Bgl}$ II. For the reporter assay, cells in each well were transfected with $0.05 \mu \mathrm{g}$ HMGCR promoter, $0.01 \mu \mathrm{g}$ TK as control, and $0.25 \mu \mathrm{g}$ c-Myc expression vectors. After $24 \mathrm{~h}$ later, cells were harvested using the lysis buffer, and the reporter activity were measured using a dual-luciferase kit (Promega Corporation) according to the manufacturer's protocols.

Immunoprecipitation assay. Caes 17 cells $\left(6 \times 10^{5}\right)$ were seeded into a $10 \mathrm{~cm}$ dish. After $24 \mathrm{~h}$, the cells were lysed with immunoprecipitation lysis buffer (9806; Cell Signaling Technology, Inc.). The cell lysates were incubated with Flag $(1: 1,000$; ab205606; Abcam) and c-Myc (1:500; 12189; Cell Signaling Technology, Inc.) primary antibodies at $4^{\circ} \mathrm{C}$ overnight. Protein A Sepharose CL-4B antibody purification resin (GE Healthcare Life Sciences, Little Chalfont, UK) were added to the supernatant and incubated for $4 \mathrm{~h}$ at $4^{\circ} \mathrm{C}$. The beads were washed three times using lysis buffer (0.05\% Tween-20). The bound protein was immunoprecipitated and analyzed by $8 \%$ SDS-PAGE (Sangon, Shanghai). The blots were examined using Bio-Rad image system.
Chromatin immunoprecipitation (ChIP) assay. ChIP assays were performed according to the manufacturer's protocol (Cell Signaling Technology, Inc.). KYSE180 cells were cross-linked with $1 \%$ formaldehyde for $10 \mathrm{~min}$ at room temperature. Subsequently, 1.0 M glycine was added to a final concentration of $125 \mathrm{mM}$ to terminate the cross-linking reaction via incubation for $5 \mathrm{~min}$ at room temperature. The cells were collected, resuspended and sonicated $(100 \mathrm{~Hz})$ six times for $5 \mathrm{sec}$ with the 1 min interval between each sonication, to shear the chromatin into 200-to 1,000-bp fragments; one-third of the lysate was used as the input following cross-link reversal, phenol/chloroform extraction and ethanol precipitation. The remaining two-thirds of the lysate were subjected to immunoprecipitation with an anti-Myc antibody $(1: 1,000 ; 12189)$ or IgG $(1: 1,000$; 14708; both Cell Signaling Technology, Inc.) at $4^{\circ} \mathrm{C}$ overnight. The immunoprecipitated complexes were collected using Protein G-Sepharose beads. The precipitates were sequentially subjected to cross-link reversal in $300 \mathrm{mM} \mathrm{NaCl}$ at $65^{\circ} \mathrm{C}$ for $8 \mathrm{~h}$, phenol/chloroform (1:1 mixture) extraction to remove the protein by centrifugation at $12,000 \mathrm{x} \mathrm{g}$ and $4^{\circ} \mathrm{C}$ for $20 \mathrm{~min}$ and $75 \%$ ethanol precipitation on ice for $4 \mathrm{~h}$. Following centrifugation $(12,000 \times \mathrm{g}$ for $15 \mathrm{~min})$, the supernatant was removed, and the DNA was resuspended in $40 \mu \mathrm{l}$ double-distilled water for qPCR analysis (2X SYBR-Green mixture, Takara Biotechnology Co., Ltd.). The thermocycling conditions were: $95^{\circ} \mathrm{C}$ for $2 \mathrm{~min}$; then 45 cycles of $94^{\circ} \mathrm{C}$ for $15 \mathrm{sec}, 55^{\circ} \mathrm{C}$ for $15 \mathrm{sec}$ and $68^{\circ} \mathrm{C}$ for $30 \mathrm{sec}$. The primers were: F, 5'-CAAGGT CGGGAGTGATGATG-3' and R, 5'-TTCCTGTGCGAACCT TAC-3'. 18S was used as an internal control. The expression of SREBP2 was expressed as Ct (18S-SREBP2).

Statistical analysis. The results are presented as the mean \pm standard deviation. The experiments were repeated three times. Differences between values were statistically analyzed using a Student's t-test or one-way analysis of variance (ANOVA) using SPSS 15.0 (SPSS, Inc., Chicago, IL, USA). Turkey was used as the post hoc test for ANOVA. $\mathrm{P}<0.05$ was considered to indicate a statistically significant difference.

\section{Results}

SREBP2 expression is increased in ESCC. Our previous study demonstrated that HMGCR was upregulated in ESCC, and statins, inhibitors of HMGCR, inhibited the growth and migration of ESCC cells (13). SREBP2 is the master regulator of HMGCR. Therefore, the expression profile of SREBP2 in ESCC tissues was investigated in the present study. The mRNA expression levels of SREBP2 in 52 ESCC tissues and paired non-cancerous tissues were detected; as presented in Fig. 1A, significantly increased SREBP2 mRNA expression levels were identified in the ESCC tissues compared with the non-cancerous tissues. In addition, the protein expression levels of SREBP2 in ESCC tissues were analyzed via immunohistochemistry and western blotting. SREBP2 protein expression levels were markedly increased in ESCC tissues compared with normal samples (Fig. 1B and C). Furthermore, SREBP2 expression in normal esophageal epithelial cells (Het-1A) and ESCC cells (KYSE150, KYSE180, Eca109 and Caes17) was investigated. As presented in Fig. 1D, decreased SREBP2 expression was detected in the normal Het-1A 


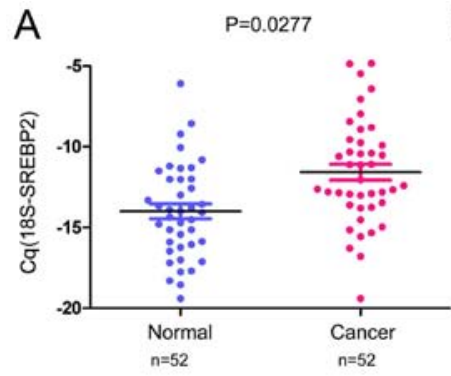

C

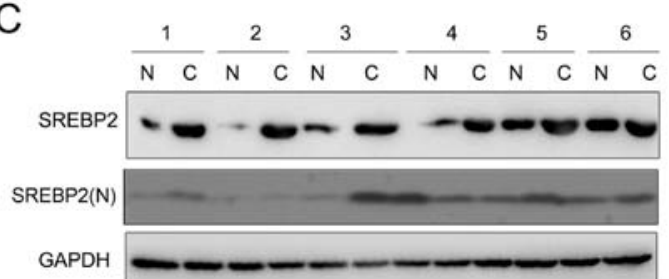

B

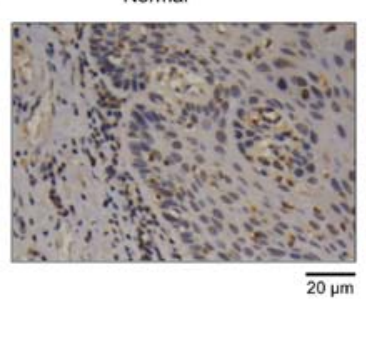

Cancer

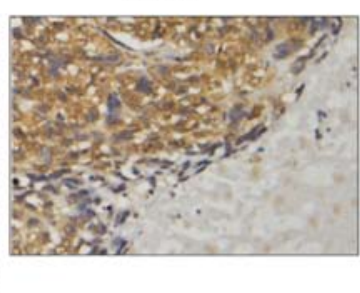

D

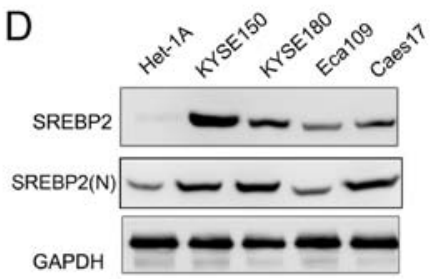

Figure 1. SREBP2 is upregulated in ESCC. (A) SREBP2 mRNA expression levels in ESCC tissues and paired non-cancerous tissues were examined by reverse transcription-quantitative polymerase chain reaction. 18S rRNA was used as an internal control. The Cq values (18S-SREBP2) were plotted. (B) SREBP2 protein expression levels in ESCC tissues and paired non-cancerous tissues were examined by immunohistochemistry. (C) Total SREBP2 and SREBP2(N) protein expression levels in ESCC tissues and paired non-cancerous tissues were examined by western blot analysis. (D) Total SREBP2 and SREBP2(N) protein expression levels in normal esophageal epithelial cells and ESCC cells were examined by western blot analysis. C, cancer; N, normal; SREBP2, sterol regulatory element-binding protein 2; SREBP2(N), N-terminal of SREBP2; ESCC, esophageal squamous cell carcinoma.

cells. The N-terminus of SREBP2 [SREBP2(N)] serves as a transcriptional factor following cleavage (16). Therefore, the expression levels of the active form of SREBP2 in ESCC tissues and cell lines were additionally detected. As presented in Fig. 1C and D, SREBP2(N) was markedly upregulated in ESCC cell lines with the exception of the Eca109 cells; however, only a few ESCC tissue samples exhibited upregulated SREBP2(N) expression levels. These data suggested that SREBP2 is upregulated in ESCC.

SREBP2 promotes ESCC cell viability, colony formation and migration. To study the biological functions of SREBP2 in ESCC, SREBP2 was overexpressed in KYSE180 and Caes17 cells, which had low expression levels of SREBP2 (Fig. 1D, Fig. 2A). SREBP2 overexpression significantly increased the KYSE180 and Caes17 cell migration and invasion in Boyden chamber assays and Transwell assays, respectively (Fig. 2B and C). Additionally, SREBP2 significantly promoted the growth and colony formation of KYSE180 and Caes17 cells in liquid culture and on the soft agar compared with the empty vector, P23 (Fig. 2D and E). Lovastatin (10 mg/l), an HMGCR inhibitor (17), significantly decreased the promoting effects of SREBP2 on the anchorage-independent growth of Caes17 and KYSE180 cells (Fig. 2E). Upregulated HMGCR expression levels were observed in Caes17 and KYSE180 cells overexpressing SREBP2; however, expression levels of HMGCR were decreased in cells treated with lovastatin (Fig. 2E). To investigate the effects of HMGCR expression on the malignant phenotype associated with SREBP2, HMGCR expression was downregulated in the present study. As presented in Fig. 2F, knockdown of HMGCR expression inhibited the anchorage-independent growth of KYSE180 cells compared with the control cells. These results suggested that SREBP2 promoted ESCC cell tumorigenesis by enhancing HMGCR activity.
To further examine the functions of endogenous SREBP2, the expression of SREBP2 was downregulated in Caes17 and KYSE180 cells (Fig. 3A). As presented in Fig. 3B and C, SREBP2 knockdown significantly inhibited the anchorage-independent growth and migration of ESCC cells compared with the control (Fig. $3 \mathrm{~B}$ and $\mathrm{C} ; \mathrm{P}<0.01$ ). Collectively, these results demonstrated that SREBP2 may promote ESCC cell growth and migration.

SREBP2 interacts with $c-M y c$ in ESCC cells. The present study screened the binding partners of SREBP2 in ESCC cells via mass spectrometry (data not shown). c-Myc was identified as a potential binding partner for SREBP2. Therefore, the interaction between c-Myc and SREBP2 was investigated via GST pull-down assays. As presented in Fig. 4A, the fusion protein GST-Myc bound to SREBP2 in Caes17 cells. In addition, exogenously expressed c-Myc [hemagglutinin (HA)-Myc] and SREBP2 (Flag-SREBP2) co-transfected with Lipofectamine $^{\circledR} 2000$ (Invitrogen; Thermo Fisher Scientific, Inc.) formed a complex in Caes17 cells (Fig. 4B). Furthermore, endogenously expressed c-Myc and SREBP2 interacted with each other (Fig. 4C). Collectively, these data suggested that c-Myc and SREBP2 form a complex.

C-Myc is essentialfor SREBP2-induced HMGCR expression. To further investigate the function of the interaction between c-Myc and SREBP2, the effects of c-Myc on HMGCR expression were studied. Overexpression of c-Myc significantly increased the mRNA expression levels of HMGCR in Caes17 and KYSE180 cells compared with the control (Fig. 5A; $\mathrm{P}<0.01$ ), whereas, knockdown of c-Myc expression significantly inhibited HMGCR expression compared with the control (Fig. 5B; P<0.01). Additionally, whether c-Myc activates HMGCR promoter activity was investigated in the present study. As presented in Fig. 5C, c-Myc significantly activated the HMGCR promoter in a dose-dependent manner 
A

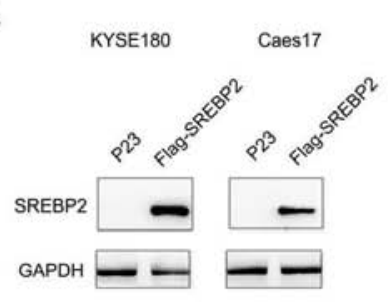

B

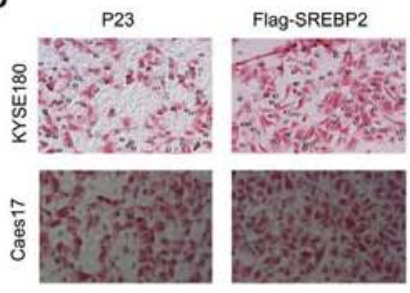

KYSE180 Caes17

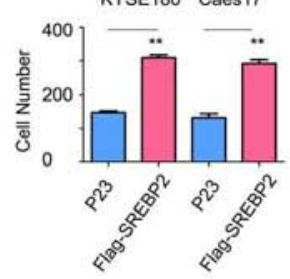

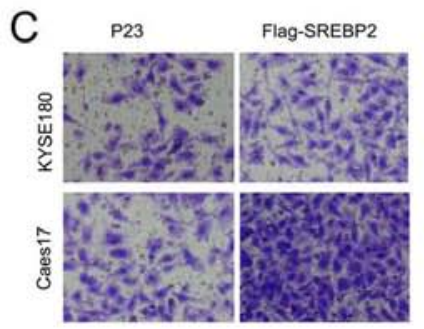
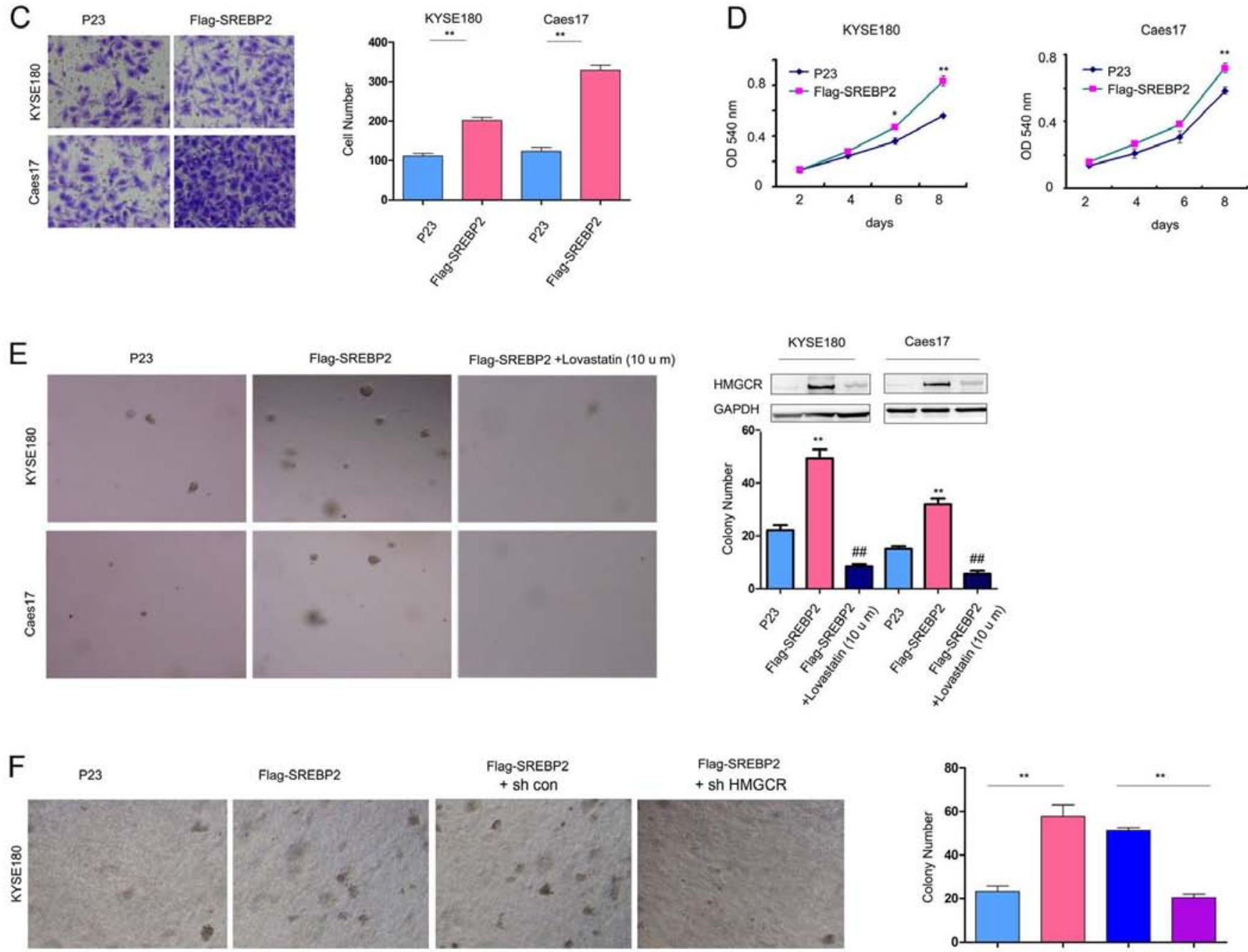

Flag-SREBP2

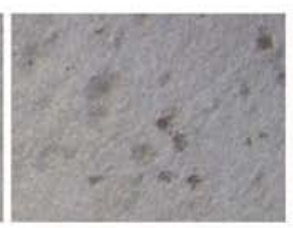

Flag-SREBP2 + sh con

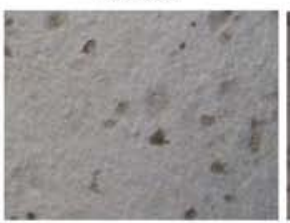

Flag-SREBP2 + sh HMGCR

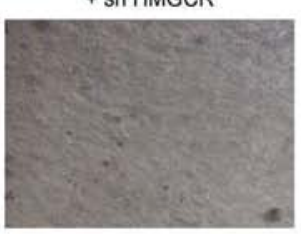

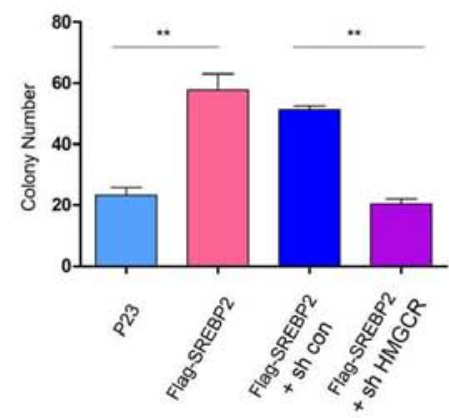

HMGCR

SREBP2

GAPDH
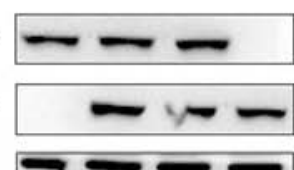

Figure 2. SREBP2 promotes ESCC cell viability, invasion and migration. (A) Constitutive SREBP2 expression in Caes17 and KYSE180 cells. Effects of SREBP2 on ESCC cell (B) migration and (C) invasion were evaluated using Boyden chamber and Transwell assays. Magnification, x20. (D) Effects of SREBP2 on ESCC cell viability were evaluated via MTT assays. (E) Effects of SREBP2 on the anchorage-independent growth of ESCC cells were evaluated by a soft agar assay; treatment with lovastatin rescued the effects of SREBP2. Magnification, x20. (F) Roles of HMGCR in the anchorage-independent growth of ESCC cells induced by SREBP2. Magnification, $\mathrm{x} 20 .{ }^{*} \mathrm{P}<0.05,{ }^{* *} \mathrm{P}<0.01$ vs. respective $\mathrm{P} 23 ;{ }^{\# \#} \mathrm{P}<0.01$ vs. respective Flag-SREBP2. sh, short hairpin RNA; SREBP2, sterol regulatory element-binding protein 2; ESCC, esophageal squamous cell carcinoma; HMGCR, 3-hydroxy 3-methylglutaryl coenzyme A reductase; OD, optical density.

compared with the control group $(\mathrm{P}<0.01)$ in KYSE180 cells. Additionally, c-Myc was determined to directly bind the
HMGCR promoter via ChIP assays (Fig. 5D). Collectively, these data suggested that c-Myc induced HMGCR expression. 


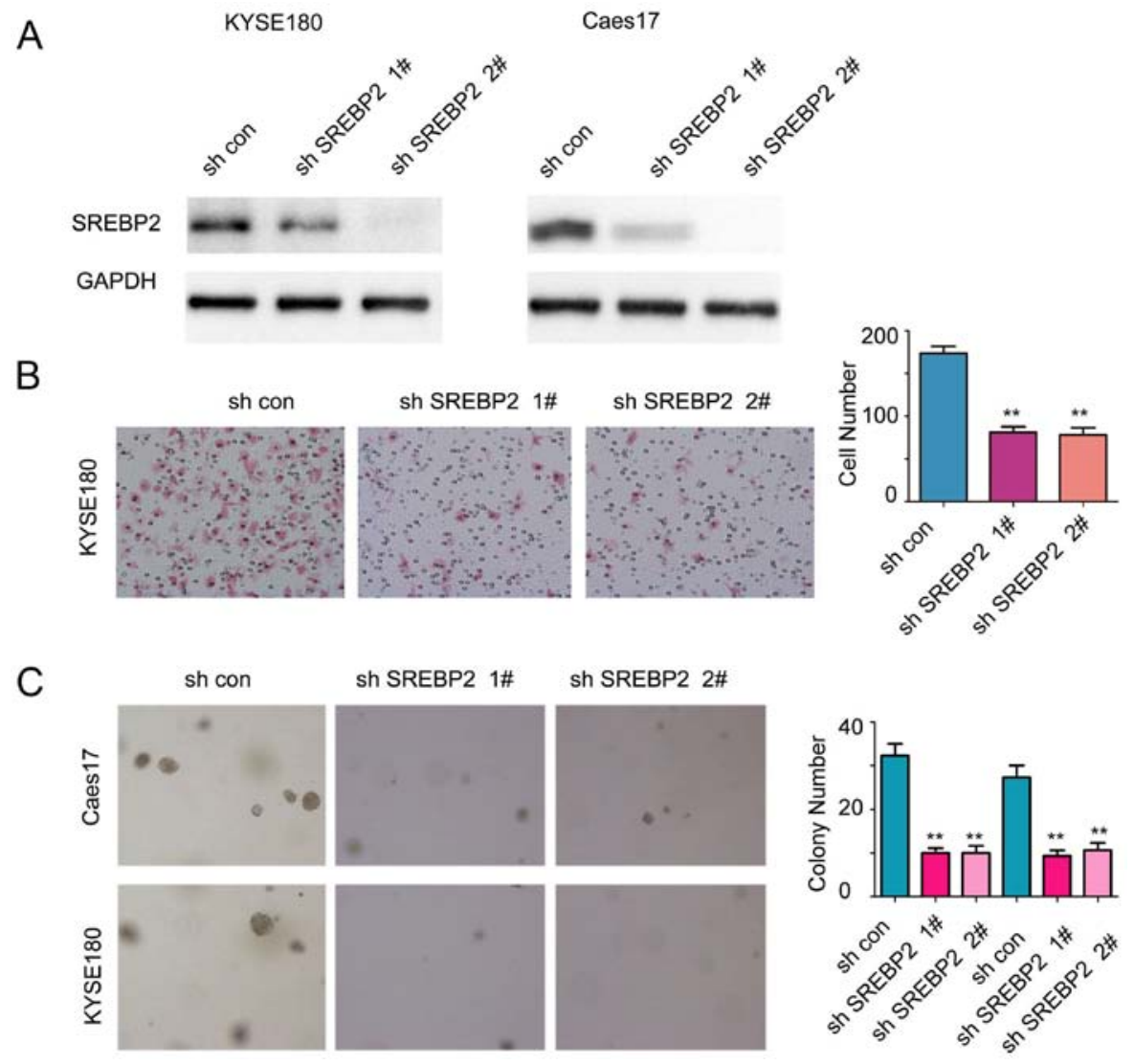

Figure 3. Downregulation of SREBP2 inhibits ESCC cell growth and migration. (A) Knockdown of SREBP2 expression in ESCC cells. (B) Effects of SREBP2 knockdown on ESCC cell migration were evaluated using Boyden chamber assays. Magnification, x20. (C) Effects of SREBP2 knockdown on the anchorage-independent growth of ESCC cells were evaluated by soft agar assays. Magnification, $\mathrm{x} 20 .{ }^{{ }^{*}} \mathrm{P}<0.01 \mathrm{vs}$. respective sh con. Con, control; sh, short hairpin RNA; SREBP2, sterol regulatory element-binding protein 2; ESCC, esophageal squamous cell carcinoma.

A
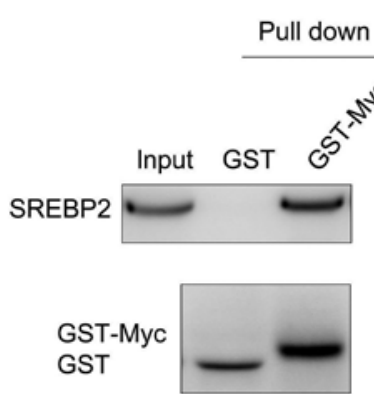

C

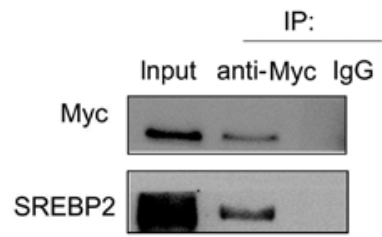

B

Caes17

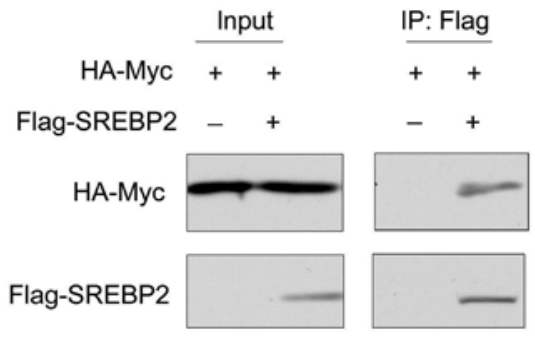

Figure 4. SREBP2 interacts with c-Myc in ESCC cells. (A) A GST pull-down assay was performed to examine the interaction between c-Myc and SREBP2. (B) IP assays were performed to examine the interaction between exogenously expressed c-Myc and SREBP2. (C) Immunoprecipitation assays were performed to examine the interaction between endogenously expressed c-Myc and SREBP2. GST, glutathione S-transferase; HA, human influenza hemagglutinin; SREBP2, sterol regulatory element-binding protein 2; ESCC, esophageal squamous cell carcinoma; IP, immunoprecipitation.

Based on the interaction between c-Myc and SREBP2, the present study subsequently investigated whether $\mathrm{c}-\mathrm{Myc}$ and SREBP2 cooperate to regulate HMGCR expression. As presented in Fig. 5E, downregulation of c-Myc impaired 
A

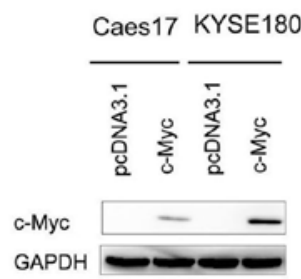

Caes 17

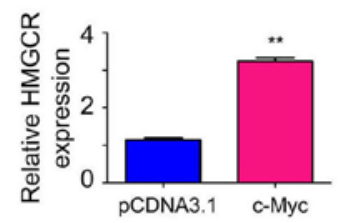

C

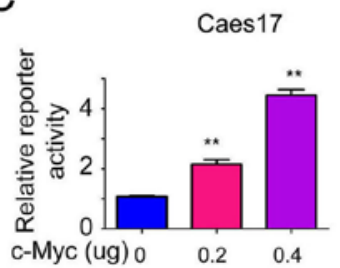

E

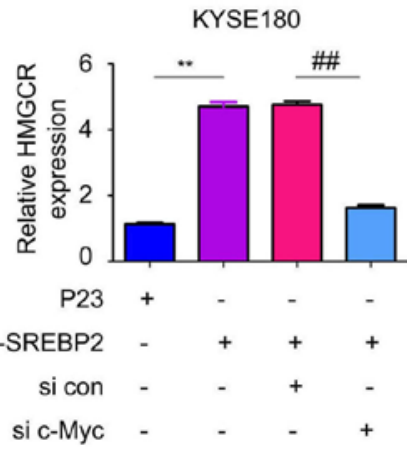

B

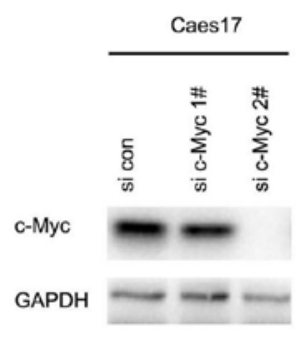

Caes 17
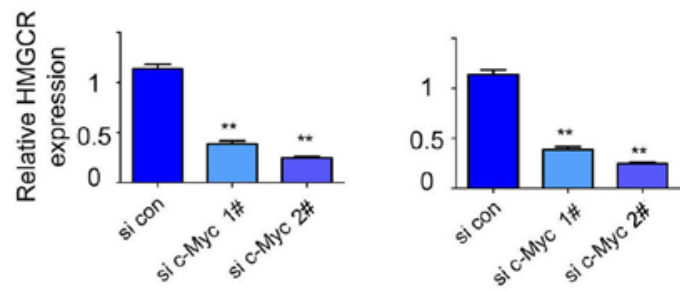

KYSE180

D

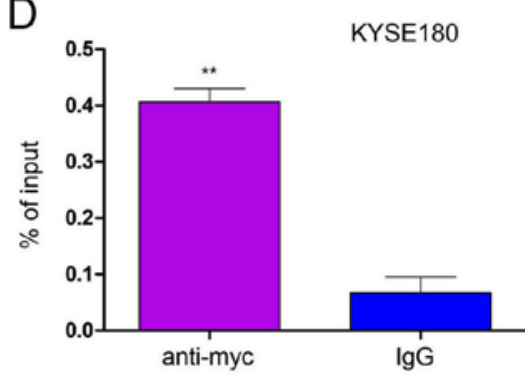

Figure 5. C-Myc promotes HMGCR expression. (A) c-Myc overexpression upregulated HMGCR mRNA expression levels. ${ }^{* *} \mathrm{P}<0.01$ vs. respective c-Myc. (B) Knockdown of c-Myc downregulated HMGCR mRNA expression levels. ${ }^{* *} \mathrm{P}<0.01$ vs. respective si con. (C) c-Myc activated the HMGCR promoter. ${ }^{* *} \mathrm{P}<0.01$ vs. respective $0 \mu \mathrm{g}$ c-Myc. (D) Chromatin immunoprecipitation assay reveals c-Myc binding to the HMGCR promoter. Quantitative polymerase chain reaction was performed. ${ }^{* *} \mathrm{P}<0.01$ vs. IgG. (E) c-Myc knockdown rescued the effects of SREBP2 on promoting HMGCR expression. ${ }^{* *} \mathrm{P}<0.01$; ${ }^{\# \#} \mathrm{P}<0.01$. con, control; HMGCR, 3-hydroxy 3-methylglutaryl coenzyme A reductase; si, small interfering RNA; SREBP2, sterol regulatory element-binding protein 2; IgG, immunoglobulin G.

the effects of SREBP2 in promoting HMGCR transcription. Collectively, these observations suggested that c-Myc is essential for SREBP2-induced HMGCR expression.

\section{Discussion}

Dysregulation of the MVA pathway has been demonstrated in numerous types of cancer $(8,18,19)$. In our previous study, it was demonstrated that ESCC cells were very sensitive to treatment with statin (12). HMGCR, the target of statin, is upregulated in ESCC samples and promotes epithelial cell migration and transformation (13). In the present study, it was demonstrated that the expression of SREBP2, a regulator of HMGCR, was increased in ESCC. SREBP2 promoted the migration and invasive abilities, viability and anchorage-independent growth of ESCC. Additionally, the present study demonstrated that c-Myc is a binding partner of SREBP2 and cooperates with SREBP2 to enhance the expression of HMGCR. Collectively, these results suggested that the MVA pathway may be activated in ESCC, thus making ESCC sensitive to treatment with statin. These results potentially suggest a novel therapeutic target for ESCC.

A notable result of the present study is the identification of c-Myc as a binding protein of SREBP2. Recently, numerous transcriptional factors have been determined to be binding partners of the SREBP protein family (16). For example, mutant P53 interacts with SREBP and promotes the activation of the MVA pathway in breast cancer cells (20), suggesting that the MVA pathway is altered by oncogenes and tumor suppressors. 
A number of previous studies demonstrated the overexpression of c-Myc in ESCC (21-25). Therefore, it may be hypothesized that the upregulation of c-Myc cooperated with SREBP2 to activate the MVA pathway and promote tumorigenesis. In addition, numerous previous studies demonstrated the key roles of c-Myc in cancer metabolism (26-28). The results of the present study suggested that c-Myc regulates cholesterol synthesis by interacting with SREBP2.

In summary, the present study demonstrated the oncogenic roles of SREBP2 in ESCC-associated tumorigenesis, suggesting that SREBP2 may be a therapeutic target for ESCC.

\section{Acknowledgements}

Not applicable.

\section{Funding}

The present study was supported by the National Science Foundation of China (grant no. 81401933) and Shanghai Science and Technology Committee (grant no. 13ZR1461300; Shanghai, China).

\section{Availability of data and materials}

The datasets used and/or analyzed during the current study are available from the corresponding author on reasonable request.

\section{Authors' contributions}

CZ, LF, FY and HZ made substantial contributions to the design of the present study. CZ, LF, FY and ZL performed the experiments. All authors read and approved the final manuscript.

\section{Ethics approval and consent to participate}

The present study was approved by the Institutional Ethics Committee of Shanghai Chest Hospital (Shanghai, China). All patients provided written informed consent.

\section{Patient consent for publication}

Not applicable.

\section{Competing interests}

The authors declare that they have no competing interests.

\section{References}

1. Siegel RL, Miller KD and Jemal A: Cancer statistics, 2017. CA Cancer J Clin 67: 7-30, 2017.

2. Schwartz L, Supuran CT and Alfarouk KO: The warburg effect and the hallmarks of cancer. Anticancer Agents Med Chem 17: 164-170, 2017.

3. Munkley J and Elliott DJ: Hallmarks of glycosylation in cancer. Oncotarget 7: 35478-35489, 2016.

4. Goldstein JL and Brown MS: Regulation of the mevalonate pathway. Nature 343: 425-430, 1990.

5. Landau BR and Brunengraber H: Shunt pathway of mevalonate metabolism. Methods Enzymol 110: 100-114, 1985.

6. Weinberger C: A model for farnesoid feedback control in the mevalonate pathway. Trends Endocrinol Metab 7: 1-6, 1996.
7. Bathaie SZ, Ashrafi M, Azizian M and Tamanoi F: Mevalonate pathway and human cancers. Curr Mol Pharmacol 10: 77-85, 2017.

8. Swanson KM and Hohl RJ: Anti-cancer therapy: Targeting the mevalonate pathway. Curr Cancer Drug Targets 6: 15-37, 2006.

9. Wang T, Seah S, Loh X, Chan CW, Hartman M, Goh BC and Lee SC: Simvastatin-induced breast cancer cell death and deactivation of PI3K/Akt and MAPK/ERK signalling are reversed by metabolic products of the mevalonate pathway. Oncotarget 7: 2532-2544, 2016

10. Madison BB: Srebp2: A master regulator of sterol and fatty acid synthesis. J Lipid Res 57: 333-335, 2016.

11. Ma K, Malhotra P, Soni V,Hedroug O, Annaba F, Dudeja A, Shen L, Turner JR, Khramtsova EA, Saksena S, et al: Overactivation of intestinal SREBP2 in mice increases serum cholesterol. PLoS One 9: e84221, 2014.

12. Shi J, Zhu J, Zhao H, Zhong C, Xu Z and Yao F: Mevalonate pathway is a therapeutic target in esophageal squamous cell carcinoma. Tumour Biol 34: 429-435, 2013.

13. Zhong C, Fan L, Yao F, Shi J, Fang W and Zhao H: HMGCR is necessary for the tumorigenecity of esophageal squamous cell carcinoma and is regulated by Myc. Tumour Biol 35: 4123-4129, 2014.

14. Xie JJ, Xu LY, Wu JY, Shen ZY, Zhao Q, Du ZP, Lv Z, Gu W, Pan F, Xu XE, et al: Involvement of CYR61 and CTGF in the fascin-mediated proliferation and invasiveness of esophageal squamous cell carcinomas cells. Am J Pathol 176: 939-951, 2010.

15. Livak KJ and Schmittgen TD: Analysis of relative gene expression data using real-time quantitative PCR and the 2(-Delta Delta C(T)) method. Methods 25: 402-408, 2001.

16. Mukherjee M, Basu Ball W and Das PK: Leishmania donovani activates SREBP2 to modulate macrophage membrane cholesterol and mitochondrial oxidants for establishment of infection. Int J Biochem Cell Biol 55: 196-208, 2014.

17. Kajinami K, Takekoshi N, Brousseau ME and Schaefer EJ: Pharmacogenetics of HMG-CoA reductase inhibitors: exploring the potential for genotype-based individualization of coronary heart disease management. Atherosclerosis 177: 219-234, 2004.

18. Caruso MG and Notarnicola M: Biochemical changes of mevalonate pathway in human colorectal cancer. Anticancer Res 25: 3393-3397, 2005.

19. Andela VB, Pirri M, Schwarz EM, Puzas EJ, O'Keefe RJ, Rosenblatt JD and Rosier RN: The mevalonate synthesis pathway as a therapeutic target in cancer. Clin Orthop Relat Res (415 Suppl): S59-S66, 2003.

20. Freed-Pastor WA, Mizuno H, Zhao X, Langerød A, Moon SH, Rodriguez-Barrueco R, Barsotti A, Chicas A, Li W, Polotskaia A, et al: Mutant p53 disrupts mammary tissue architecture via the mevalonate pathway. Cell 148: 244-258, 2012.

21. Gomes TS, Noguti J, Forones NM, Lima FO, Dobo C, Fernandes Junior JA, Oshima CT and Ribeiro DA: Correlation analysis of c-myc, p21(WAF/CIP1), p53, C-erbB-2 and COX-2 proteins in esophageal squamous cell carcinoma. Pathol Res Pract 209: 6-9, 2013.

22. Brown J, Bothma H, Veale R and Willem P: Genomic imbalances in esophageal carcinoma cell lines involve Wnt pathway genes. World J Gastroenterol 17: 2909-2923, 2011.

23. Wang W, Xue L and Wang P: Prognostic value of $\beta$-catenin, c-myc, and cyclin D1 expressions in patients with esophageal squamous cell carcinoma. Med Oncol 28: 163-169, 2011.

24. Ishizuka T, Tanabe C, Sakamoto H, Aoyagi K, Maekawa M, Matsukura N, Tokunaga A, Tajiri T, Yoshida T, Terada M and Sasaki H: Gene amplification profiling of esophageal squamous cell carcinomas by DNA array CGH. Biochem Biophys Res Commun 296: 152-155, 2002.

25. Mandard AM, Hainaut P and Hollstein M: Genetic steps in the development of squamous cell carcinoma of the esophagus. Mutat Res 462: 335-342, 2000.

26. Shukla SK, Gunda V, Abrego J, Haridas D, Mishra A, Souchek J, Chaika NV, Yu F, Sasson AR, Lazenby AJ, et al: MUC16-mediated activation of mTOR and c-Myc reprograms pancreatic cancer metabolism. Oncotarget 6: 19118-19131, 2015.

27. Miller DM, Thomas SD, Islam A, Muench D and Sedoris K: c-Myc and cancer metabolism. Clin Cancer Res 18: 5546-5553, 2012.

28. Gordan JD, Thompson CB and Simon MC: HIF and c-Myc: Sibling rivals for control of cancer cell metabolism and proliferation. Cancer cell 12: 108-113, 2007.

This work is licensed under a Creative Commons Attribution-NonCommercial-NoDerivatives 4.0 International (CC BY-NC-ND 4.0) License. 